\title{
Light tetraquark states with the exotic quantum number $J^{P C}=3^{-+}$
}

\author{
Niu Su, ${ }^{1,2}$ Rui-Rui Dong, ${ }^{1,2}$ Hua-Xing Chen $\odot,{ }^{1, *}$ Wei Chen $\odot,{ }^{3, \dagger}$ and Er-Liang Cui $\oplus^{4, \$}$ \\ ${ }^{1}$ School of Physics, Southeast University, Nanjing 210094, China \\ ${ }^{2}$ School of Physics, Beihang University, Beijing 100191, China \\ ${ }^{3}$ School of Physics, Sun Yat-Sen University, Guangzhou 510275, China \\ ${ }^{4}$ College of Science, Northwest A\&F University, Yangling 712100, China
}

(Received 22 November 2020; accepted 15 February 2021; published 3 March 2021)

\begin{abstract}
We apply the method of QCD sum rules to study the $s q \bar{s} \bar{q}$ tetraquark states with the exotic quantum number $J^{P C}=3^{-+}$, and extract mass of the lowest-lying state to be $2.33_{-0.16}^{+0.19} \mathrm{GeV}$. To construct the relevant tetraquark currents we need to explicitly add the covariant derivative operator. Our systematical analysis on their relevant interpolating currents indicates that (a) this state well decays into the $P$-wave $\rho \phi / \omega \phi$ channel but not into the $\rho f_{2}(1525) / \omega f_{2}(1525) / \phi f_{2}(1270)$ channels, and (b) it well decays into the $K^{*}(892) \bar{K}_{2}^{*}(1430)$ channel but not into the $P$-wave $K^{*}(892) \bar{K}^{*}(892)$ channel.

DOI: 10.1103/PhysRevD.103.054006
\end{abstract}

\section{INTRODUCTION}

There have been many candidates of exotic hadrons observed in particle experiments, which cannot be well explained in the traditional quark model [1-10]. Many of them still have "traditional" quantum numbers that traditional $\bar{q} q$ mesons and $q q q$ baryons can also have. This makes them not so easy to be clearly identified as exotic hadrons. However, there exist some "exotic" quantum numbers that traditional hadrons cannot have, such as the spin-parity quantum numbers $J^{P C}=0^{--}, 0^{+-}, 1^{-+}$, $2^{+-}, 3^{-+}$, etc. These exotic quantum numbers are of particular interest, because the hadrons with such quantum numbers cannot be explained as traditional hadrons any more. Such hadrons are definitely exotic hadrons, whose possible interpretations are tetraquark states [11-17], hybrid states [18-23], glueballs [24-26], etc. Note that these different exotic structures may mix together, and there would exist various possibilities whenever there is found a state in experiment with some exotic quantum number.

Among the above exotic quantum numbers, the hybrid states of $J^{P C}=1^{-+}$have been extensively studied, since they are predicted to be the lightest hybrid states [18] and there are some experimental evidence on their existence [27-29]. The light tetraquark states of $J^{P C}=1^{-+}$have also

\footnotetext{
*hxchen@seu.edu.cn

†chenwei29@mail.sysu.edu.cn

*erliang.cui@nwafu.edu.cn
}

Published by the American Physical Society under the terms of the Creative Commons Attribution 4.0 International license. Further distribution of this work must maintain attribution to the author(s) and the published article's title, journal citation, and DOI. Funded by SCOAP . been studied in Refs. [11,12] using the method of QCD sum rules, and their masses and possible decay channels were predicted there for both isospin- 0 and isospin- 1 states. Later, the same QCD sum rule method was applied to extensively study light tetraquark states of $J^{P C}=0^{--}$in Refs. [13-15], and those of $J^{P C}=0^{+-}$in Refs. [16,17].

In this paper we shall investigate the exotic quantum number $J^{P C}=3^{-+}$, and the other one $J^{P C}=2^{+-}$will be studied in the future. We shall investigate the light $q s \bar{q} \bar{s}$ ( $q=$ up/down and $s=$ strange) tetraquark states with such a quantum number. They may exist in the energy region around $2.0 \mathrm{GeV}$. With a large amount of the $J / \psi$ sample, the BESIII Collaboration is carefully examining the physics happening in this energy region [30-36]. The Belle-II [37] and GlueX [38] experiments also do this. Hence, these states are potential exotic hadrons to be observed in future experiments. There has not been any theoretical study directly on this subject. In Ref. [39] the authors used the one-boson-exchange model to study the $D^{*} \bar{D}_{2}^{*}$ molecular state of $J^{P C}=3^{-+}$. They found that the isoscalar $(I=0)$ state has the most attractive potential, suggesting that this $D^{*} \bar{D}_{2}^{*}$ molecular state of $J^{P C}=3^{-+}$may exist, and the $K^{*}(892) \bar{K}_{2}^{*}(1430)$ molecular state of $J^{P C}=3^{-+}$might also exist. Besides, there was a Lattice QCD study on the $3^{-+}$glueball, but this was done forty years ago [40].

In this paper we shall investigate the $q s \bar{q} \bar{s}$ tetraquark state with the exotic quantum number $J^{P C}=3^{-+}$using the method of QCD sum rules. Recently, we have applied this method to study the $s s \bar{s} \bar{s}$ tetraquark states of $J^{P C}=1^{ \pm-}$in Refs. [41-43]. In the present study we shall improve it by explicitly adding the covariant derivative operator in order to construct the $q s \bar{q} \bar{s}$ tetraquark currents of $J^{P C}=3^{-+}$. This will be discussed in detail in the next section. 
This paper is organized as follows. In Sec. II, we systematically construct the $q s \bar{q} \bar{s}$ tetraquark currents with the exotic quantum number $J^{P C}=3^{-+}$. Then we use them to perform QCD sum rule analyses in Sec. III, and perform numerical analyses in Sec. IV. The results are summarized and discussed in Sec. V, where we discuss their special decay behavior.

\section{INTERPOLATING CURRENTS}

In this section we construct the $q s \bar{q} \bar{s}$ ( $q=$ up/down and $s=$ strange) tetraquark currents with the exotic quantum number $J^{P C}=3^{-+}$. This quantum number is exotic, and cannot be simply composed by using one quark and one antiquark. Moreover, we cannot use only two quarks and two antiquarks without derivatives, and two quarks and two antiquarks together with at least one derivative are necessary to reach such a quantum number.

Besides, the $s s \bar{s} \bar{s}$ tetraquark currents of $J^{P C}=3^{-+}$ cannot be constructed using two quarks and two antiquarks with just one derivative; in the present study we shall not investigate the $q q \bar{q} \bar{q}$ tetraquark currents, since the widths of the $q s \bar{q} \bar{s}$ tetraquark states (if they exist) are probably narrower, making them easier of being observed.

First let us consider the diquark-antidiquark $[q s][\bar{q} \bar{s}]$ construction. In principle, the derivative can be either inside the diquark/antidiquark field or between the diquark and antidiquark fields, i.e.,

$$
\begin{gathered}
\eta=\left[q_{a}^{T} C \Gamma_{1} \stackrel{\leftrightarrow}{D}_{\alpha} s_{b}\right]\left(\bar{q}_{c} \Gamma_{2} C \bar{s}_{d}^{T}\right), \\
\eta^{\prime}=\left(q_{a}^{T} C \Gamma_{1} s_{b}\right)\left[\bar{q}_{c} \Gamma_{2} C \stackrel{\leftrightarrow}{D}_{\alpha} \bar{s}_{d}^{T}\right], \\
\eta^{\prime \prime}=\left[\left(q_{a}^{T} C \Gamma_{3} s_{b}\right) \stackrel{\leftrightarrow}{D}_{\alpha}\left(\bar{q}_{c} \Gamma_{4} C \bar{s}_{d}^{T}\right)\right],
\end{gathered}
$$

where $\left[X \stackrel{\leftrightarrow}{D}_{\alpha} Y\right]=X\left[D_{\alpha} Y\right]-\left[D_{\alpha} X\right] Y$, with the covariant derivative $D_{\alpha}=\partial_{\alpha}+i g_{s} A_{\alpha} ; a \cdots d$ are color indices, and the sum over repeated indices is taken; $\Gamma_{1,2,3,4}$ are Dirac matrices. However, we find that only the former construction can reach the quantum number $J^{P C}=3^{-+}$.

Altogether we find six nonvanishing diquark-antidiquark currents of $J^{P C}=3^{-+}$:

$$
\begin{aligned}
\eta_{\alpha_{1} \alpha_{2} \alpha_{3}}^{1}= & \epsilon^{a b e} \epsilon^{c d e} \times \mathcal{S}\left\{\left[q_{a}^{T} C \gamma_{\alpha_{1}}{\stackrel{\leftrightarrow}{\alpha_{3}}}_{\alpha_{3}} s_{b}\right]\left(\bar{q}_{c} \gamma_{\alpha_{2}} C \bar{s}_{d}^{T}\right)\right. \\
& \left.+\left(q_{a}^{T} C \gamma_{\alpha_{1}} s_{b}\right)\left[\bar{q}_{c} \gamma_{\alpha_{2}} C \stackrel{\leftrightarrow}{D}_{\alpha_{3}} \bar{s}_{d}^{T}\right]\right\}, \\
\eta_{\alpha_{1} \alpha_{2} \alpha_{3}}^{2}= & \left(\delta^{a c} \delta^{b d}+\delta^{a d} \delta^{b c}\right) \times \mathcal{S}\left\{\left[q_{a}^{T} C \gamma_{\alpha_{1}} \stackrel{\leftrightarrow}{D}_{\alpha_{3}} s_{b}\right]\left(\bar{q}_{c} \gamma_{\alpha_{2}} C \bar{s}_{d}^{T}\right)\right. \\
& \left.+\left(q_{a}^{T} C \gamma_{\alpha_{1}} s_{b}\right)\left[\bar{q}_{c} \gamma_{\alpha_{2}} C \stackrel{\leftrightarrow}{D}_{\alpha_{3}} \bar{s}_{d}^{T}\right]\right\},
\end{aligned}
$$

$$
\begin{aligned}
& \eta_{\alpha_{1} \alpha_{2} \alpha_{3}}^{3}=\epsilon^{a b e} \epsilon^{c d e} \times \mathcal{S}\left\{\left[q_{a}^{T} C \gamma_{\alpha_{1}} \gamma_{5} \stackrel{\leftrightarrow}{D}_{\alpha_{3}} s_{b}\right]\left(\bar{q}_{c} \gamma_{\alpha_{2}} \gamma_{5} C \bar{s}_{d}^{T}\right)\right. \\
&\left.+\left(q_{a}^{T} C \gamma_{\alpha_{1}} \gamma_{5} s_{b}\right)\left[\bar{q}_{c} \gamma_{\alpha_{2}} \gamma_{5} C \stackrel{\leftrightarrow}{D}_{\alpha_{3}} \bar{s}_{d}^{T}\right]\right\}, \\
& \eta_{\alpha_{1} \alpha_{2} \alpha_{3}}^{4}=\left(\delta^{a c} \delta^{b d}+\delta^{a d} \delta^{b c}\right) \\
& \times \mathcal{S}\left\{\left[q_{a}^{T} C \gamma_{\alpha_{1}} \gamma_{5} \stackrel{\leftrightarrow}{D}_{\alpha_{3}} s_{b}\right]\left(\bar{q}_{c} \gamma_{\alpha_{2}} \gamma_{5} C \bar{s}_{d}^{T}\right)\right. \\
&\left.+\left(q_{a}^{T} C \gamma_{\alpha_{1}} \gamma_{5} s_{b}\right)\left[\bar{q}_{c} \gamma_{\alpha_{2}} \gamma_{5} C \stackrel{\leftrightarrow}{D}_{\alpha_{3}} \bar{s}_{d}^{T}\right]\right\},
\end{aligned}
$$

$$
\begin{aligned}
\eta_{\alpha_{1} \alpha_{2} \alpha_{3}}^{5}=\epsilon^{a b e} & \epsilon^{c d e} \times g^{\mu \nu} \mathcal{S}\left\{\left[q_{a}^{T} C \sigma_{\alpha_{1} \mu} \stackrel{\leftrightarrow}{D}_{\alpha_{3}} s_{b}\right]\left(\bar{q}_{c} \sigma_{\alpha_{2} \nu} C \bar{s}_{d}^{T}\right)\right. \\
+( & \left.\left.q_{a}^{T} C \sigma_{\alpha_{1} \mu} s_{b}\right)\left[\bar{q}_{c} \sigma_{\alpha_{2} \nu} C \stackrel{\leftrightarrow}{D}_{\alpha_{3}} \bar{s}_{d}^{T}\right]\right\} \\
\eta_{\alpha_{1} \alpha_{2} \alpha_{3}}^{6}= & \left(\delta^{a c} \delta^{b d}+\delta^{a d} \delta^{b c}\right) \\
& \times g^{\mu \nu} \mathcal{S}\left\{\left[q_{a}^{T} C \sigma_{\alpha_{1} \mu} \stackrel{\leftrightarrow}{D}_{\alpha_{3}} s_{b}\right]\left(\bar{q}_{c} \sigma_{\alpha_{2} \nu} C \bar{s}_{d}^{T}\right)\right. \\
& \left.+\left(q_{a}^{T} C \sigma_{\alpha_{1} \mu} s_{b}\right)\left[\bar{q}_{c} \sigma_{\alpha_{2} \nu} C \stackrel{\leftrightarrow}{D}_{\alpha_{3}} \bar{s}_{d}^{T}\right]\right\}
\end{aligned}
$$

where $\mathcal{S}$ denotes symmetrization and subtracting the trace terms in the set $\left\{\alpha_{1} \alpha_{2} \alpha_{3}\right\}$. Three of them $\eta_{\alpha_{1} \alpha_{2} \alpha_{3}}^{1,3,5}$ have the antisymmetric color structure $(q s)_{\overline{\mathbf{3}}_{C}}(\bar{q} \bar{s})_{\mathbf{3}_{C}}$, and the other three $\eta_{\alpha_{1} \alpha_{2} \alpha_{3}}^{2,4,6}$ have the symmetric color structure $(q s)_{\mathbf{6}_{C}}(\bar{q} \bar{s})_{\overline{\mathbf{6}}_{C}}$. Considering that the diquark fields $s_{a}^{T} C \gamma_{\mu} s_{b} / s_{a}^{T} C \gamma_{\mu} \gamma_{5} s_{b} / s_{a}^{T} C \sigma_{\mu \nu} s_{b}$ have the quantum numbers $J^{P}=1^{+} / 1^{-} / 1^{ \pm}$, respectively, the first current $\eta_{\alpha_{1} \alpha_{2} \alpha_{3}}^{1}$ has the most stable internal structure and may lead to the best sum rule result.

Besides the above diquark-antidiquark currents, we can construct six color-singlet-color-singlet mesonic-mesonic currents of $J^{P C}=3^{-+}$:

$$
\begin{aligned}
\xi_{\alpha_{1} \alpha_{2} \alpha_{3}}^{1}= & \mathcal{S}\left\{\left(\bar{q}_{a} \gamma_{\alpha_{1}} q_{a}\right) \stackrel{\leftrightarrow}{D}_{\alpha_{3}}\left(\bar{s}_{b} \gamma_{\alpha_{2}} s_{b}\right)\right\} \\
\xi_{\alpha_{1} \alpha_{2} \alpha_{3}}^{2}= & \mathcal{S}\left\{\left(\bar{q}_{a} \gamma_{\alpha_{1}} \gamma_{5} q_{a}\right) \stackrel{\leftrightarrow}{D}_{\alpha_{3}}\left(\bar{s}_{b} \gamma_{\alpha_{2}} \gamma_{5} s_{b}\right)\right\} \\
\xi_{\alpha_{1} \alpha_{2} \alpha_{3}}^{3}= & g^{\mu \nu} \mathcal{S}\left\{\left(\bar{q}_{a} \sigma_{\alpha_{1} \mu} q_{a}\right) \stackrel{\leftrightarrow}{D}_{\alpha_{3}}\left(\bar{s}_{b} \sigma_{\alpha_{2} \nu} s_{b}\right)\right\} \\
\xi_{\alpha_{1} \alpha_{2} \alpha_{3}}^{4}= & \mathcal{S}\left\{\left[\bar{q}_{a} \gamma_{\alpha_{1}} \stackrel{\leftrightarrow}{D}_{\alpha_{3}} s_{a}\right]\left(\bar{s}_{b} \gamma_{\alpha_{2}} q_{b}\right)\right. \\
& \left.\left(\bar{q}_{a} \gamma_{\alpha_{1}} s_{a}\right)\left[\bar{s}_{b} \gamma_{\alpha_{2}} \stackrel{\leftrightarrow}{D}_{\alpha_{3}} q_{b}\right]\right\} \\
\xi_{\alpha_{1} \alpha_{2} \alpha_{3}}^{5}= & \mathcal{S}\left\{\left[\bar{q}_{a} \gamma_{\alpha_{1}} \gamma_{5} \stackrel{\leftrightarrow}{D}_{\alpha_{3}} s_{a}\right]\left(\bar{s}_{b} \gamma_{\alpha_{2}} \gamma_{5} q_{b}\right)\right. \\
& \left.-\left(\bar{q}_{a} \gamma_{\alpha_{1}} \gamma_{5} s_{a}\right)\left[\bar{s}_{b} \gamma_{\alpha_{2}} \gamma_{5} \stackrel{\leftrightarrow}{D}_{\alpha_{3}} q_{b}\right]\right\} \\
\xi_{\alpha_{1} \alpha_{2} \alpha_{3}}^{6}= & g^{\mu \nu} \mathcal{S}\left\{\left[\bar{q}_{a} \sigma_{\alpha_{1} \mu} \stackrel{\leftrightarrow}{D}_{\alpha_{3}} s_{a}\right]\left(\bar{s}_{b} \sigma_{\alpha_{2} \nu} q_{b}\right)\right. \\
& \left.-\left(\bar{q}_{a} \sigma_{\alpha_{1} \mu} s_{a}\right)\left[\bar{s}_{b} \sigma_{\alpha_{2} \nu} \stackrel{\leftrightarrow}{D}_{\alpha_{3}} q_{b}\right]\right\}
\end{aligned}
$$

The former three $\xi_{\alpha_{1} \alpha_{2} \alpha_{3}}^{1,2,3}$ have the quark combination $[\bar{q} q][\bar{s} s]$, and the derivatives are between the two 
quark-antiquark pairs; the latter three $\xi_{\alpha_{1} \alpha_{2} \alpha_{3}}^{4,5}$ have the quark combination $[\bar{q} s][\bar{s} q]$, and the derivatives are inside the quark-antiquark pairs. This difference is useful when investigating their decay properties, which will be discussed in Sec. V.

We can further construct six color-octet-color-octet mesonic-mesonic currents, which can be related to the above color-singlet-color-singlet mesonic-mesonic currents through the Fierz transformation. Moreover, we can apply the Fierz transformation to derive the relations between diquark-antidiquark and mesonic-mesonic currents:

$$
\left(\begin{array}{l}
\eta_{\alpha_{1} \alpha_{2} \alpha_{3}}^{1} \\
\eta_{\alpha_{1} \alpha_{2} \alpha_{3}}^{2} \\
\eta_{\alpha_{1} \alpha_{2} \alpha_{3}}^{3} \\
\eta_{\alpha_{1} \alpha_{2} \alpha_{3}}^{4} \\
\eta_{\alpha_{1} \alpha_{2} \alpha_{3}}^{5} \\
\eta_{\alpha_{1} \alpha_{2} \alpha_{3}}^{6}
\end{array}\right)=\left(\begin{array}{cccccc}
-\frac{1}{2} & \frac{1}{2} & \frac{1}{2} & -\frac{1}{2} & \frac{1}{2} & \frac{1}{2} \\
-\frac{1}{2} & \frac{1}{2} & \frac{1}{2} & \frac{1}{2} & -\frac{1}{2} & -\frac{1}{2} \\
\frac{1}{2} & -\frac{1}{2} & \frac{1}{2} & -\frac{1}{2} & \frac{1}{2} & -\frac{1}{2} \\
\frac{1}{2} & -\frac{1}{2} & \frac{1}{2} & \frac{1}{2} & -\frac{1}{2} & \frac{1}{2} \\
1 & 1 & 0 & 1 & 1 & 0 \\
1 & 1 & 0 & -1 & -1 & 0
\end{array}\right)\left(\begin{array}{l}
\xi_{\alpha_{1} \alpha_{2} \alpha_{3}}^{1} \\
\xi_{\alpha_{1} \alpha_{2} \alpha_{3}}^{2} \\
\xi_{\alpha_{1} \alpha_{2} \alpha_{3}}^{3} \\
\xi_{\alpha_{1} \alpha_{2} \alpha_{3}}^{4} \\
\xi_{\alpha_{1} \alpha_{2} \alpha_{3}}^{5} \\
\xi_{\alpha_{1} \alpha_{2} \alpha_{3}}^{6}
\end{array}\right) .
$$

Therefore, these two constructions are equivalent, and in the following we shall only use $\eta_{\alpha_{1} \alpha_{2} \alpha_{3}}^{1 \cdots 6}$ to perform QCD sum rule analyses. Note that this equivalence is just between diquark-antidiquark and mesonic-mesonic currents, while compact diquark-antidiquark tetraquark states and weakly bound meson-meson molecular states are totally different. To exactly describe them, one needs nonlocal interpolating currents, but we are still not capable of using such currents to perform QCD sum rule analyses.

\section{QCD SUM RULE ANALYSIS}

In this section we use the currents $\eta_{\alpha_{1} \alpha_{2} \alpha_{3}}^{1 \cdots 6}$ to perform QCD sum rule analyses. We assume that they couple to some exotic state $X$ through

$$
\left\langle 0\left|\eta_{\alpha_{1} \alpha_{2} \alpha_{3}}\right| X\right\rangle=f_{X} \epsilon_{\alpha_{1} \alpha_{2} \alpha_{3}}
$$

where $f_{X}$ is the decay constant and $\epsilon_{\alpha_{1} \alpha_{2} \alpha_{3}}$ is the traceless and symmetric polarization tensor, satisfying the following:

$$
\epsilon_{\alpha_{1} \alpha_{2} \alpha_{3}} \epsilon_{\beta_{1} \beta_{2} \beta_{3}}^{*}=\mathcal{S}^{\prime}\left[\tilde{g}_{\alpha_{1} \beta_{1}} \tilde{g}_{\alpha_{2} \beta_{2}} \tilde{g}_{\alpha_{3} \beta_{3}}\right] .
$$

In this expression $\tilde{g}_{\mu \nu}=g_{\mu \nu}-q_{\mu} q_{\nu} / q^{2}$, and $\mathcal{S}^{\prime}$ denotes symmetrization and subtracting the trace terms in the sets $\left\{\alpha_{1} \alpha_{2} \alpha_{3}\right\}$ and $\left\{\beta_{1} \beta_{2} \beta_{3}\right\}$.

Based on Eq. (16), we study the two-point correlation function

$$
\begin{aligned}
& \Pi_{\alpha_{1} \alpha_{2} \alpha_{3}, \beta_{1} \beta_{2} \beta_{3}}\left(q^{2}\right) \\
& \quad \equiv i \int d^{4} x e^{i q x}\left\langle 0\left|\mathbf{T}\left[\eta_{\alpha_{1} \alpha_{2} \alpha_{3}}(x) \eta_{\beta_{1} \beta_{2} \beta_{3}}^{\dagger}(0)\right]\right| 0\right\rangle \\
& \quad=(-1)^{J} \mathcal{S}^{\prime}\left[\tilde{g}_{\alpha_{1} \beta_{1}} \tilde{g}_{\alpha_{2} \beta_{2}} \tilde{g}_{\alpha_{3} \beta_{3}}\right] \Pi\left(q^{2}\right),
\end{aligned}
$$

at both hadron and quark-gluon levels.

At the hadron level we use the dispersion relation to express Eq. (18) as

$$
\Pi\left(q^{2}\right)=\int_{4 m_{s}^{2}}^{\infty} \frac{\rho(s)}{s-q^{2}-i \varepsilon} d s
$$

where $\rho(s)$ is the spectral density. Then we parametrize it using one pole dominance for the ground state $X$ and a continuum contribution:

$$
\begin{aligned}
\rho(s) & \equiv \sum_{n} \delta\left(s-M_{n}^{2}\right)\langle 0|\eta| n\rangle\left\langle n\left|\eta^{\dagger}\right| 0\right\rangle \\
& =f_{X}^{2} \delta\left(s-M_{X}^{2}\right)+\text { continuum. }
\end{aligned}
$$

At the quark-gluon level we insert $\eta_{\alpha_{1} \alpha_{2} \alpha_{3}}^{1 \cdots 6}$ into Eq. (18) and calculate it using the method of operator product expansion (OPE). After performing the Borel transformation to Eq. (18) at both hadron and quark-gluon levels, we can approximate the continuum using the spectral density above a threshold value $s_{0}$, and obtain the sum rule equation

$$
\Pi\left(s_{0}, M_{B}^{2}\right) \equiv f_{X}^{2} e^{-M_{X}^{2} / M_{B}^{2}}=\int_{4 m_{s}^{2}}^{s_{0}} e^{-s / M_{B}^{2}} \rho(s) d s .
$$

We can use it to further evaluate $M_{X}$, the mass of $X$, through,

$$
\begin{aligned}
M_{X}^{2}\left(s_{0}, M_{B}\right) & =\frac{\frac{\partial}{\partial\left(-1 / M_{B}^{2}\right)} \Pi\left(s_{0}, M_{B}^{2}\right)}{\Pi\left(s_{0}, M_{B}^{2}\right)} \\
& =\frac{\int_{4 m_{s}^{2}}^{s_{0}} e^{-s / M_{B}^{2}} S \rho(s) d s}{\int_{4 m_{s}^{2}}^{s_{0}} e^{-s / M_{B}^{2}} \rho(s) d s} .
\end{aligned}
$$

In the present study we have calculated OPEs up to the tenth dimension, including the perturbative term, the strange quark mass, the gluon condensate, the quark condensate, the quark-gluon mixed condensate, and their combinations: 


$$
\begin{aligned}
& \Pi_{11}=\int_{4 m_{s}^{2}}^{s_{0}}\left[\frac{s^{5}}{691200 \pi^{6}}-\frac{m_{s}^{2} s^{4}}{14336 \pi^{6}}+\left(-\frac{179\left\langle g_{s}^{2} G G\right\rangle}{5806080 \pi^{6}}+\frac{m_{s}^{4}}{2016 \pi^{6}}-\frac{m_{s}\langle\bar{q} q\rangle}{720 \pi^{4}}+\frac{m_{s}\langle\bar{s} s\rangle}{1512 \pi^{4}}\right) s^{3}\right. \\
& +\left(\frac{37\left\langle g_{s}^{2} G G\right\rangle m_{s}^{2}}{122880 \pi^{6}}-\frac{91 m_{s}\left\langle g_{s} \bar{q} \sigma G q\right\rangle}{30720 \pi^{4}}+\frac{m_{s}^{3}\langle\bar{q} q\rangle}{80 \pi^{4}}-\frac{m_{s}^{3}\langle\bar{s} s\rangle}{240 \pi^{4}}+\frac{\langle\bar{q} q\rangle\langle\bar{s} s\rangle}{60 \pi^{2}}\right) s^{2} \\
& +\left(-\frac{\left\langle g_{s}^{2} G G\right\rangle m_{s}^{4}}{18432 \pi^{6}}+\frac{3 m_{s}^{3}\left\langle g_{s} \bar{q} \sigma G q\right\rangle}{256 \pi^{4}}+\frac{5\left\langle g_{s}^{2} G G\right\rangle m_{s}\langle\bar{q} q\rangle}{3456 \pi^{4}}-\frac{7\left\langle g_{s}^{2} G G\right\rangle m_{s}\langle\bar{s} s\rangle}{8640 \pi^{4}}+\frac{5\left\langle g_{s} \bar{q} \sigma G q\right\rangle\langle\bar{s} s\rangle}{288 \pi^{2}}-\frac{m_{s}^{2}\langle\bar{q} q\rangle\langle\bar{s} s\rangle}{12 \pi^{2}}\right. \\
& \left.+\frac{5\langle\bar{q} q\rangle\left\langle g_{s} \bar{s} \sigma G s\right\rangle}{288 \pi^{2}}\right) s+\left(\frac{\left\langle g_{s}^{2} G G\right\rangle m_{s}\left\langle g_{s} \bar{q} \sigma G q\right\rangle}{4608 \pi^{4}}-\frac{m_{s}^{2}\left\langle g_{s} \bar{q} \sigma G q\right\rangle\langle\bar{q} q\rangle}{12 \pi^{2}}+\frac{\left\langle g_{s}^{2} G G\right\rangle m_{s}^{3}\langle\bar{s} s\rangle}{13824 \pi^{4}}-\frac{3 m_{s}^{2}\left\langle g_{s} \bar{q} \sigma G q\right\rangle\langle\bar{s} s\rangle}{128 \pi^{2}}\right. \\
& \left.\left.-\frac{\left\langle g_{s}^{2} G G\right\rangle\langle\bar{q} q\rangle\langle\bar{s} s\rangle}{324 \pi^{2}}+\frac{17\left\langle g_{s} \bar{q} \sigma G q\right\rangle\left\langle g_{s} \bar{s} \sigma G s\right\rangle}{3456 \pi^{2}}-\frac{m_{s}^{2}\langle\bar{q} q\rangle\left\langle g_{s} \bar{s} \sigma G s\right\rangle}{576 \pi^{2}}\right)\right] e^{-s / M^{2}} d s \\
& +\left(-\frac{m_{s}^{2}\left\langle g_{s} \bar{q} \sigma G q\right\rangle^{2}}{24 \pi^{2}}+\frac{2 m_{s}\left\langle g_{s} \bar{q} \sigma G q\right\rangle\langle\bar{q} q\rangle\langle\bar{s} s\rangle}{9}\right) \\
& \Pi_{22}=\int_{4 m_{s}^{2}}^{s_{0}}\left[\frac{s^{5}}{345600 \pi^{6}}-\frac{m_{s}^{2}}{7168 \pi^{6}} s^{4}+\left(-\frac{199\left\langle g_{s}^{2} G G\right\rangle}{5806080 \pi^{6}}+\frac{m_{s}^{4}}{1008 \pi^{6}}-\frac{m_{s}\langle\bar{q} q\rangle}{360 \pi^{4}}+\frac{m_{s}\langle\bar{s} s\rangle}{756 \pi^{4}}\right) s^{3}\right. \\
& +\left(\frac{41 m_{s}^{2}\left\langle g_{s}^{2} G G\right\rangle}{122880 \pi^{6}}-\frac{239 m_{s}\left\langle g_{s} \bar{q} \sigma G q\right\rangle}{30720 \pi^{4}}+\frac{m_{s}^{3}\langle\bar{q} q\rangle}{40 \pi^{4}}-\frac{m_{s}^{3}\langle\bar{s} s\rangle}{120 \pi^{4}}+\frac{\langle\bar{q} q\rangle\langle\bar{s} s\rangle}{30 \pi^{2}}\right) s^{2} \\
& +\left(-\frac{5 m_{s}^{4}\left\langle g_{s}^{2} G G\right\rangle}{18432 \pi^{6}}+\frac{7 m_{s}^{3}\left\langle g_{s} \bar{q} \sigma G q\right\rangle}{256 \pi^{4}}-\frac{5 m_{s}\langle\bar{q} q\rangle\left\langle g_{s}^{2} G G\right\rangle}{3456 \pi^{2}}-\frac{m_{s}\langle\bar{s} s\rangle\left\langle g_{s}^{2} G G\right\rangle}{1080 \pi^{4}}+\frac{13\langle\bar{s} s\rangle\left\langle g_{s} \bar{q} \sigma G q\right\rangle}{288 \pi^{2}}-\frac{m_{s}^{2}\langle\bar{s} s\rangle\langle\bar{q} q\rangle}{6 \pi^{2}}\right. \\
& \left.+\frac{13\langle\bar{q} q\rangle\left\langle g_{s} \bar{s} \sigma G s\right\rangle}{288 \pi^{2}}\right) s+\left(\frac{49\left\langle g_{s} \bar{q} \sigma G q\right\rangle\left\langle g_{s} \bar{s} \sigma G s\right\rangle}{3456 \pi^{2}}-\frac{5 m_{s}^{2}\langle\bar{q} q\rangle\left\langle g_{s} \bar{s} \sigma G s\right\rangle}{576 \pi^{2}}-\frac{m_{s}\left\langle g_{s}^{2} G G\right\rangle\left\langle g_{s} \bar{q} \sigma G q\right\rangle}{4608 \pi^{4}}-\frac{m_{s}^{2}\langle\bar{q} q\rangle\left\langle g_{s} \bar{q} \sigma G q\right\rangle}{6 \pi^{2}}\right. \\
& \left.\left.+\frac{5 m_{s}^{3}\langle\bar{s} s\rangle\left\langle g_{s}^{2} G G\right\rangle}{13824 \pi^{4}}-\frac{7 m_{s}^{2}\langle\bar{s} s\rangle\left\langle g_{s} \bar{q} \sigma G q\right\rangle}{128 \pi^{2}}+\frac{\langle\bar{q} q\rangle\langle\bar{s} s\rangle\left\langle g_{s}^{2} G G\right\rangle}{324 \pi^{2}}\right)\right] e^{-s / M_{B}^{2}} d s \\
& +\left(-\frac{m_{s}^{2}\left\langle g_{s} \bar{q} \sigma G q\right\rangle^{2}}{12 \pi^{2}}+\frac{4 m_{s}\langle\bar{s} s\rangle\langle\bar{q} q\rangle\left\langle g_{s} \bar{q} \sigma G q\right\rangle}{9}\right) \\
& \Pi_{33}=\int_{4 m_{s}^{2}}^{s_{0}}\left[+\frac{s^{5}}{691200 \pi^{6}}-\frac{m_{s}^{2} s^{4}}{14336 \pi^{6}}+\left(-\frac{179\left\langle g_{s}^{2} G G\right\rangle}{5806080 \pi^{6}}+\frac{m_{s}^{4}}{2016 \pi^{6}}+\frac{m_{s}\langle\bar{q} q\rangle}{720 \pi^{4}}+\frac{m_{s}\langle\bar{s} s\rangle}{1512 \pi^{4}}\right) s^{3}\right. \\
& +\left(\frac{37\left\langle g_{s}^{2} G G\right\rangle m_{s}^{2}}{122880 \pi^{6}}+\frac{91 m_{s}\left\langle g_{s} \bar{q} \sigma G q\right\rangle}{30720 \pi^{4}}-\frac{m_{s}^{3}\langle\bar{q} q\rangle}{80 \pi^{4}}-\frac{m_{s}^{3}\langle\bar{s} s\rangle}{240 \pi^{4}}-\frac{\langle\bar{q} q\rangle\langle\bar{s} s\rangle}{60 \pi^{2}}\right) s^{2} \\
& +\left(-\frac{\left\langle g_{s}^{2} G G\right\rangle m_{s}^{4}}{18432 \pi^{6}}-\frac{3 m_{s}^{3}\left\langle g_{s} \bar{q} \sigma G q\right\rangle}{256 \pi^{4}}-\frac{5\left\langle g_{s}^{2} G G\right\rangle m_{s}\langle\bar{q} q\rangle}{3456 \pi^{4}}-\frac{7\left\langle g_{s}^{2} G G\right\rangle m_{s}\langle\bar{s} s\rangle}{8640 \pi^{4}}-\frac{5\left\langle g_{s} \bar{q} \sigma G q\right\rangle\langle\bar{s} s\rangle}{288 \pi^{2}}+\frac{m_{s}^{2}\langle\bar{q} q\rangle\langle\bar{s} s\rangle}{12 \pi^{2}}\right. \\
& \left.-\frac{5\langle\bar{q} q\rangle\left\langle g_{s} \bar{s} \sigma G s\right\rangle}{288 \pi^{2}}\right) s+\left(-\frac{\left\langle g_{s}^{2} G G\right\rangle m_{s}\left\langle g_{s} \bar{q} \sigma G q\right\rangle}{4608 \pi^{4}}-\frac{m_{s}^{2}\left\langle g_{s} \bar{q} \sigma G q\right\rangle\langle\bar{q} q\rangle}{12 \pi^{2}}+\frac{\left\langle g_{s}^{2} G G\right\rangle m_{s}^{3}\langle\bar{s} s\rangle}{13824 \pi^{4}}+\frac{3 m_{s}^{2}\left\langle g_{s} \bar{q} \sigma G q\right\rangle\langle\bar{s} s\rangle}{128 \pi^{2}}\right. \\
& \left.\left.+\frac{\left\langle g_{s}^{2} G G\right\rangle\langle\bar{q} q\rangle\langle\bar{s} s\rangle}{324 \pi^{2}}-\frac{17\left\langle g_{s} \bar{q} \sigma G q\right\rangle\left\langle g_{s} \bar{s} \sigma G s\right\rangle}{3456 \pi^{2}}+\frac{m_{s}^{2}\langle\bar{q} q\rangle\left\langle g_{s} \bar{s} \sigma G s\right\rangle}{576 \pi^{2}}\right)\right] e^{-s / M^{2}} d s \\
& +\left(-\frac{m_{s}^{2}\left\langle g_{s} \bar{q} \sigma G q\right\rangle^{2}}{24 \pi^{2}}+\frac{2 m_{s}\left\langle g_{s} \bar{q} \sigma G q\right\rangle\langle\bar{q} q\rangle\langle\bar{s} s\rangle}{9}\right)
\end{aligned}
$$




$$
\begin{aligned}
& \Pi_{44}=\int_{4 m_{s}^{2}}^{s_{0}}\left[\frac{s^{5}}{345600 \pi^{6}}-\frac{m_{s}^{2} s^{4}}{7168 \pi^{6}}+\left(-\frac{199\left\langle g_{s}^{2} G G\right\rangle}{5806080 \pi^{6}}+\frac{m_{s}^{4}}{1008 \pi^{6}}+\frac{m_{s}\langle\bar{q} q\rangle}{360 \pi^{4}}+\frac{m_{s}\langle\bar{s} s\rangle}{756 \pi^{4}}\right) s^{3}\right. \\
& +\left(\frac{41 m_{s}^{2}\left\langle g_{s}^{2} G G\right\rangle}{122880 \pi^{6}}+\frac{239 m_{s}\left\langle g_{s} \bar{q} \sigma G q\right\rangle}{30720 \pi^{4}}-\frac{m_{s}^{3}\langle\bar{q} q\rangle}{40 \pi^{4}}-\frac{m_{s}^{3}\langle\bar{s} s\rangle}{120 \pi^{4}}-\frac{\langle\bar{q} q\rangle\langle\bar{s} s\rangle}{30 \pi^{2}}\right) s^{2} \\
& +\left(-\frac{5 m_{s}^{4}\left\langle g_{s}^{2} G G\right\rangle}{18432 \pi^{6}}-\frac{7 m_{s}^{3}\left\langle g_{s} \bar{q} \sigma G q\right\rangle}{256 \pi^{4}}+\frac{5 m_{s}\langle\bar{q} q\rangle\left\langle g_{s}^{2} G G\right\rangle}{3456 \pi^{4}}-\frac{m_{s}\langle\bar{s} s\rangle\left\langle g_{s}^{2} G G\right\rangle}{1080 \pi^{4}}-\frac{13\langle\bar{s} s\rangle\left\langle g_{s} \bar{q} \sigma G q\right\rangle}{288 \pi^{2}}+\frac{m_{s}^{2}\langle\bar{s} s\rangle\langle\bar{q} q\rangle}{6 \pi^{2}}\right. \\
& \left.-\frac{13\langle\bar{q} q\rangle\left\langle g_{s} \bar{s} \sigma G s\right\rangle}{288 \pi^{2}}\right) s+\left(\frac{m_{s}\left\langle g_{s}^{2} G G\right\rangle\left\langle g_{s} \bar{q} \sigma G q\right\rangle}{4608 \pi^{4}}+\frac{5 m_{s}^{3}\left\langle g_{s}^{2} G G\right\rangle\langle\bar{s} s\rangle}{13824 \pi^{4}}-\frac{m_{s}^{2}\langle\bar{q} q\rangle\left\langle g_{s} \bar{q} \sigma G q\right\rangle}{6 \pi^{2}}+\frac{7 m_{s}^{2}\langle\bar{s} s\rangle\left\langle g_{s} \bar{q} \sigma G q\right\rangle}{128 \pi^{2}}\right. \\
& \left.\left.-\frac{\langle\bar{s} s\rangle\langle\bar{q} q\rangle\left\langle g_{s}^{2} G G\right\rangle}{324 \pi^{2}}-\frac{49\left\langle g_{s} \bar{q} \sigma G q\right\rangle\left\langle g_{s} \bar{s} \sigma G s\right\rangle}{3456 \pi^{2}}+\frac{5 m_{s}^{2}\langle\bar{q} q\rangle\left\langle g_{s} \bar{s} \sigma G s\right\rangle}{576 \pi^{2}}\right)\right] e^{-s / M_{B}^{2}} d s \\
& +\left(\frac{4 m_{s}\langle\bar{q} q\rangle\langle\bar{s} s\rangle\left\langle g_{s} \bar{q} \sigma G q\right\rangle}{9}-\frac{m_{s}^{2}\left\langle g_{s} \bar{q} \sigma G q\right\rangle^{2}}{12 \pi^{2}}\right), \\
& \Pi_{55}=\int_{4 m_{s}^{2}}^{s_{0}}\left[+\frac{s^{5}}{345600 \pi^{6}}-\frac{m_{s}^{2} s^{4}}{7168 \pi^{6}}+\left(-\frac{73\left\langle g_{s}^{2} G G\right\rangle}{1451520 \pi^{6}}+\frac{m_{s}^{4}}{1008 \pi^{6}}+\frac{m_{s}\langle\bar{s} s\rangle}{756 \pi^{4}}\right) s^{3}+\left(\frac{\left\langle g_{s}^{2} G G\right\rangle m_{s}^{2}}{2048 \pi^{6}}-\frac{m_{s}^{3}\langle\bar{s} s\rangle}{120 \pi^{4}}\right) s^{2}\right. \\
& \left.+\left(-\frac{\left\langle g_{s}^{2} G G\right\rangle m_{s}^{4}}{11520 \pi^{6}}-\frac{23\left\langle g_{s}^{2} G G\right\rangle m_{s}\langle\bar{s} s\rangle}{17280 \pi^{4}}\right) s-\frac{m_{s}^{2}\left\langle g_{s} \bar{q} \sigma G q\right\rangle\langle\bar{s} s\rangle}{6 \pi^{2}}+\frac{\left\langle g_{s}^{2} G G\right\rangle m_{s}^{3}\langle\bar{s} s\rangle}{6912 \pi^{4}}\right] e^{-s / M_{B}^{2}} d s \\
& +\left(-\frac{m_{s}^{2}\left\langle g_{s} \bar{q} \sigma G q\right\rangle^{2}}{12 \pi^{2}}+\frac{4 m_{s}\left\langle g_{s} \bar{q} \sigma G q\right\rangle\langle\bar{q} q\rangle\langle\bar{s} s\rangle}{9}\right), \\
& \Pi_{66}=\int_{4 m_{s}^{2}}^{s_{0}}\left[\frac{s^{5}}{172800 \pi^{6}}-\frac{m_{s}^{2} s^{4}}{3584 \pi^{6}}+\left(-\frac{25\left\langle g_{s}^{2} G G\right\rangle}{290304 \pi^{6}}+\frac{m_{s}^{4}}{504 \pi^{6}}+\frac{m_{s}\langle\bar{s} s\rangle}{378 \pi^{4}}\right) s^{3}+\left(\frac{9 m_{s}^{2}\left\langle g_{s}^{2} G G\right\rangle}{10240 \pi^{6}}-\frac{m_{s}^{3}\langle\bar{s} s\rangle}{60 \pi^{4}}\right) s^{2}\right. \\
& \left.+\left(-\frac{m_{s}^{4}\left\langle g_{s}^{2} G G\right\rangle}{2304 \pi^{6}}-\frac{43 m_{s}\left\langle g_{s}^{2} G G\right\rangle\langle\bar{s} s\rangle}{17280 \pi^{4}}\right) s-\frac{m_{s}^{2}\left\langle g_{s} \bar{q} \sigma G q\right\rangle\langle\bar{q} q\rangle}{3 \pi^{2}}+\frac{5 m_{s}^{3}\left\langle g_{s}^{2} G G\right\rangle\langle\bar{s} s\rangle}{6912 \pi^{4}}\right] e^{-s / M_{B}^{2}} d s \\
& +\left(-\frac{m_{s}^{2}\left\langle g_{s} \bar{q} \sigma G q\right\rangle^{2}}{6 \pi^{2}}+\frac{8 m_{s}\left\langle g_{s} \bar{q} \sigma G q\right\rangle\langle\bar{q} q\rangle\langle\bar{s} s\rangle}{9}\right) \text {. }
\end{aligned}
$$

Based on these expressions, we shall perform numerical analyses in the next section.

\section{NUMERICAL ANALYSES}

In this section we use the sum rules given in Eqs. (23)-(28) to perform numerical analyses. The following values are used for various quark and gluon parameters $[1,44-50]$ :

$$
\begin{aligned}
m_{s}(2 \mathrm{GeV}) & =96_{-4}^{+8} \mathrm{MeV}, \\
\left\langle g_{s}^{2} G G\right\rangle & =(0.48 \pm 0.14) \mathrm{GeV}^{4}, \\
\langle\bar{q} q\rangle & =-(0.240 \pm 0.010)^{3} \mathrm{GeV}^{3}, \\
\langle\bar{s} s\rangle & =(0.8 \pm 0.1) \times\langle\bar{q} q\rangle, \\
\left\langle g_{s} \bar{q} \sigma G q\right\rangle & =-M_{0}^{2} \times\langle\bar{q} q\rangle, \\
\left\langle g_{s} \bar{s} \sigma G s\right\rangle & =-M_{0}^{2} \times\langle\bar{s} s\rangle, \\
M_{0}^{2} & =(0.8 \pm 0.2) \mathrm{GeV}^{2} .
\end{aligned}
$$

To begin with, we show Eqs. (23)-(28) in Fig. 1 as functions of the threshold value $s_{0}$. We find that $\Pi_{33}\left(M_{B}^{2}, s_{0}\right)$ and $\Pi_{44}\left(M_{B}^{2}, s_{0}\right)$ are both negative when $s_{0}$ is around $6 \mathrm{GeV}^{2}$. This suggests that they are both nonphysical in this energy region, so we shall not investigate $\eta_{\alpha_{1} \alpha_{2} \alpha_{3}}^{3}$ and $\eta_{\alpha_{1} \alpha_{2} \alpha_{3}}^{4}$ any more.

The mass $M_{X}$ depends on two free parameters, the threshold value $s_{0}$ and the Borel mass $M_{B}$. To find their proper working regions, we investigate three aspects: (a) the OPE convergence, (b) the pole contribution, and (c) the mass dependence on $M_{B}$ and $s_{0}$.

Taking the current $\eta_{\alpha_{1} \alpha_{2} \alpha_{3}}^{1}$ as an example, whose sum rules are given in Eq. (23), we first investigate the convergence of OPE, which is the cornerstone of a reliable QCD sum rule analysis. We require the $D=10$ term to be less than 5\%:

$$
\text { Convergence }(\mathrm{CVG}) \equiv\left|\frac{\Pi_{11}^{D=10}\left(s_{0}, M_{B}^{2}\right)}{\Pi_{11}\left(s_{0}, M_{B}^{2}\right)}\right| \leq 5 \% .
$$



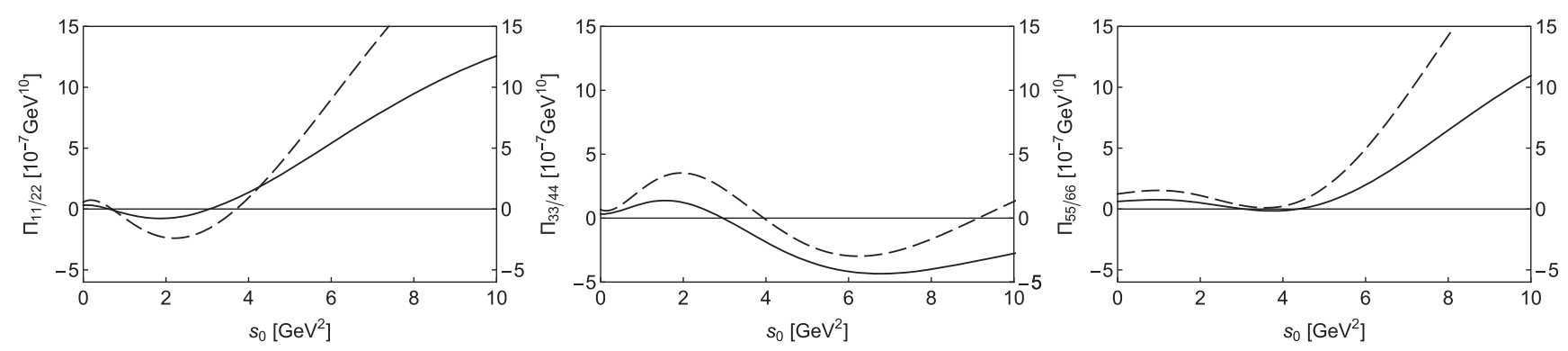

FIG. 1. The two-point correlation functions, $\Pi_{11}\left(s_{0}, M_{B}^{2}\right)$ (left-solid), $\Pi_{22}\left(s_{0}, M_{B}^{2}\right)$ (left-dashed), $\Pi_{33}\left(s_{0}, M_{B}^{2}\right)$ (middle-solid), $\Pi_{44}\left(s_{0}, M_{B}^{2}\right)$ (middle-dashed), $\Pi_{55}\left(s_{0}, M_{B}^{2}\right)$ (right-solid), and $\Pi_{66}\left(s_{0}, M_{B}^{2}\right)$ (right-dashed), as functions of the threshold value $s_{0}$. These curves are obtained by setting $M_{B}^{2}=1.4 \mathrm{GeV}^{2}$.

As shown in Fig. 2 using the solid curve, the lower bound of the Borel mass is determined to be $M_{B}^{2}>1.32 \mathrm{GeV}^{2}$, when setting $s_{0}=7.2 \mathrm{GeV}^{2}$.

Then we investigate the one-pole-dominance assumption by requiring the pole contribution (PC) to be larger than $45 \%$ :

$$
\mathrm{PC} \equiv\left|\frac{\Pi_{11}\left(s_{0}, M_{B}^{2}\right)}{\Pi_{11}\left(\infty, M_{B}^{2}\right)}\right| \geq 45 \%
$$

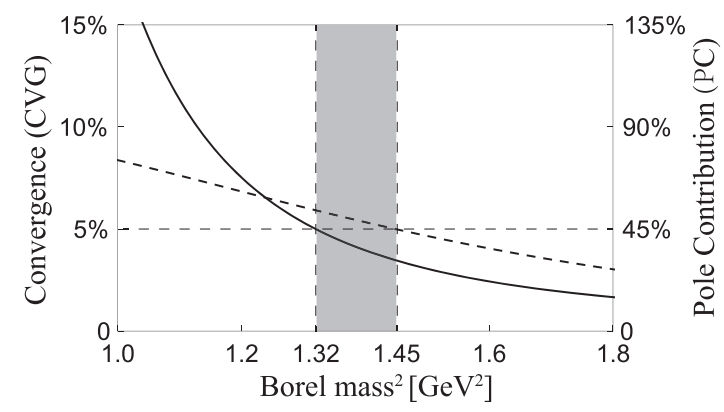

FIG. 2. CVG [solid curve, defined in Eq. (30)] and PC [dashed curve, defined in Eq. (31)] as functions of the Borel mass $M_{B}$. These curves are obtained using the current $\eta_{\alpha_{1} \alpha_{2} \alpha_{3}}^{1}$ when setting $s_{0}=7.2 \mathrm{GeV}^{2}$.

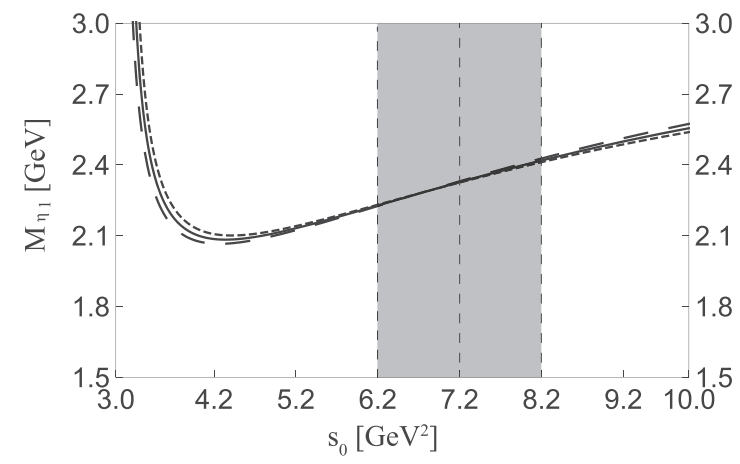

As shown in Fig. 2 using the dashed curve, the upper bound of the Borel mass is determined to be $M_{B}^{2}<1.45 \mathrm{GeV}^{2}$, when setting $s_{0}=7.2 \mathrm{GeV}^{2}$.

Altogether we obtain the Borel window to be $1.32 \mathrm{GeV}^{2}<M_{B}^{2}<1.45 \mathrm{GeV}^{2}$ when setting $s_{0}=7.2 \mathrm{GeV}^{2}$. Redoing the same procedures by changing $s_{0}$, we find that there are nonvanishing Borel windows as long as $s_{0}>6.7 \mathrm{GeV}^{2}$.

Finally, we study the mass dependence on $M_{B}$ and $s_{0}$. We show the mass $M_{X}$ in Fig. 3 with respective to these two parameters. It is stable around $s_{0} \sim 7.2 \mathrm{GeV}^{2}$, and its dependence on $M_{B}$ is weak in the Borel window $1.32 \mathrm{GeV}^{2}<M_{B}^{2}<1.45 \mathrm{GeV}^{2}$. Accordingly, we choose the working regions to be $6.2 \mathrm{GeV}^{2}<s_{0}<8.2 \mathrm{GeV}^{2}$ and $1.32 \mathrm{GeV}^{2}<M_{B}^{2}<1.45 \mathrm{GeV}^{2}$, where the mass $M_{X}$ is evaluated to be

$$
M_{\eta_{1}}=2.33_{-0.16}^{+0.19} \mathrm{GeV} .
$$

Here the central value corresponds to $s_{0}=7.2 \mathrm{GeV}^{2}$ and $M_{B}^{2}=1.38 \mathrm{GeV}^{2}$, and the uncertainty comes from $M_{B}, s_{0}$, and various quark and gluon parameters listed in Eq. (30).

Similarly, we use $\eta_{\alpha_{1} \alpha_{2} \alpha_{3}}^{2}$ to perform numerical analyses. We show the mass extracted in Fig. 4 as a function of the threshold value $s_{0}$ (left) and the Borel mass $M_{B}$ (right).

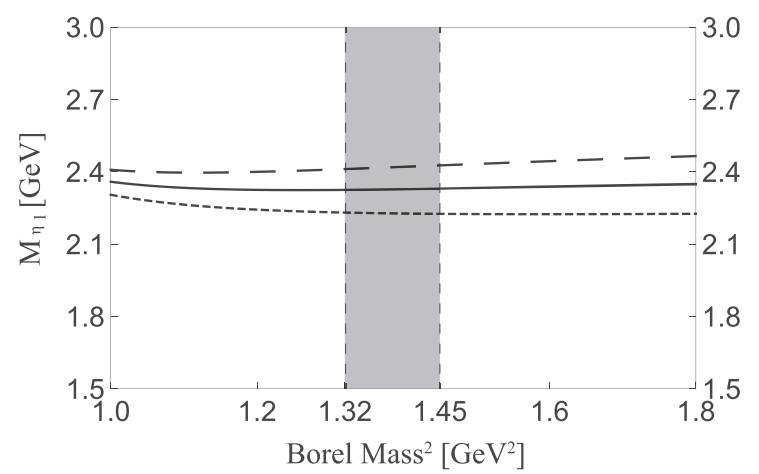

FIG. 3. Mass calculated using the current $\eta_{\alpha_{1} \alpha_{2} \alpha_{3}}^{1}$, as a function of the threshold value $s_{0}$ (left) and the Borel mass $M_{B}$ (right). In the left panel the short-dashed/solid/long-dashed curves are depicted when setting $M_{B}^{2}=1.32 / 1.38 / 1.45 \mathrm{GeV}^{2}$, respectively. In the right panel the short-dashed/solid/long-dashed curves are depicted when setting $s_{0}=6.2 / 7.2 / 8.2 \mathrm{GeV}^{2}$, respectively. 

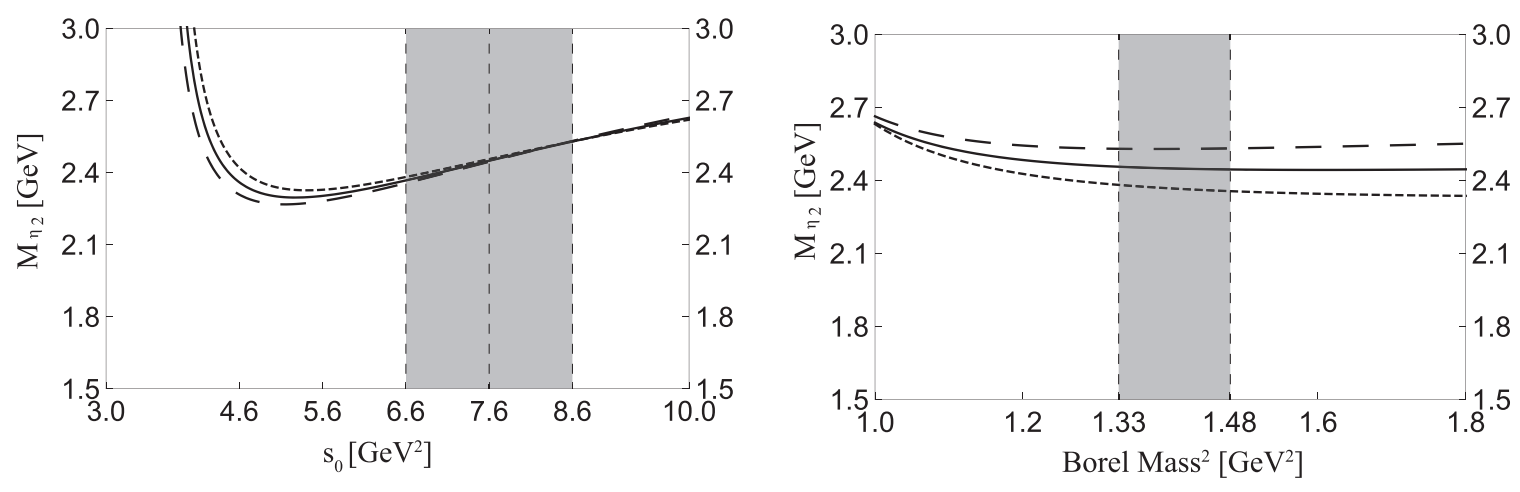

FIG. 4. Mass calculated using the current $\eta_{\alpha_{1} \alpha_{2} \alpha_{3}}^{2}$, as a function of the threshold value $s_{0}$ (left) and the Borel mass $M_{B}$ (right). In the left panel the short-dashed/solid/long-dashed curves are depicted when setting $M_{B}^{2}=1.33 / 1.40 / 1.48 \mathrm{GeV}^{2}$, respectively. In the right panel the short-dashed/solid/long-dashed curves are depicted when setting $s_{0}=6.6 / 7.6 / 8.6 \mathrm{GeV}^{2}$, respectively.

After extracting the working regions to be $6.6 \mathrm{GeV}^{2}<$ $s_{0}<8.6 \mathrm{GeV}^{2}$ and $1.33 \mathrm{GeV}^{2}<M_{B}^{2}<1.48 \mathrm{GeV}^{2}$, we obtain

$$
M_{\eta_{2}}=2.45_{-0.18}^{+0.27} \mathrm{GeV}
$$

where the central value corresponds to $s_{0}=7.6 \mathrm{GeV}^{2}$ and $M_{B}^{2}=1.40 \mathrm{GeV}^{2}$.

The same procedures are applied to analyses of the currents $\eta_{\alpha_{1} \alpha_{2} \alpha_{3}}^{5}$ and $\eta_{\alpha_{1} \alpha_{2} \alpha_{3}}^{6}$, but the masses extracted from them are significantly larger than those from $\eta_{\alpha_{1} \alpha_{2} \alpha_{3}}^{1}$ and $\eta_{\alpha_{1} \alpha_{2} \alpha_{3}}^{2}$. We summarize all the results in Table I.

It is interesting to investigate the mixing of $\eta_{\alpha_{1} \alpha_{2} \alpha_{3}}^{1}$ and $\eta_{\alpha_{1} \alpha_{2} \alpha_{3}}^{2}$, since the possibly existing physical state may have a structure much more complicated than those described by these two single currents [41-43]:

$$
\eta_{\alpha_{1} \alpha_{2} \alpha_{3}}^{\operatorname{mix}}(\theta) \equiv \cos \theta \eta_{\alpha_{1} \alpha_{2} \alpha_{3}}^{1}+\sin \theta \eta_{\alpha_{1} \alpha_{2} \alpha_{3}}^{2} .
$$

However, we find that the mass minimum is arrived just at $\theta=0^{\circ}$, that is $\eta_{\alpha_{1} \alpha_{2} \alpha_{3}}^{\operatorname{mix}}\left(0^{\circ}\right)=\eta_{\alpha_{1} \alpha_{2} \alpha_{3}}^{1}$. Hence, this mixing does not change the extracted mass, and we shall use the results extracted from the current $\eta_{\alpha_{1} \alpha_{2} \alpha_{3}}^{1}$ to draw conclusions in the next section.

TABLE I. Masses extracted from the currents $\eta_{\alpha_{1} \alpha_{2} \alpha_{3}}^{1,2,5,}$.

\begin{tabular}{lcccc}
\hline \hline Currents & $M_{B}^{2}\left[\mathrm{GeV}^{2}\right]$ & $s_{0}\left[\mathrm{GeV}^{2}\right]$ & Pole [\%] & Mass [GeV] \\
\hline$\eta_{\alpha_{1} \alpha_{2} \alpha_{3}}^{1}$ & $1.32-1.45$ & $7.2 \pm 1.0$ & $44.9-53.3$ & $2.33_{-0.16}^{+0.19}$ \\
$\eta_{\alpha_{1} \alpha_{2} \alpha_{3}}^{2}$ & $1.33-1.48$ & $7.6 \pm 1.0$ & $45.1-54.1$ & $2.45_{-0.18}^{+0.27}$ \\
$\eta_{\alpha_{1} \alpha_{2} \alpha_{3}}^{5}$ & $1.46-1.60$ & $9.6 \pm 1.0$ & $45.1-53.4$ & $2.72_{-0.12}^{+0.11}$ \\
$\eta_{\alpha_{1} \alpha_{2} \alpha_{3}}^{6}$ & $1.45-1.58$ & $9.4 \pm 1.0$ & $45.2-53.1$ & $2.67_{-0.12}^{+0.11}$ \\
\hline \hline
\end{tabular}

\section{SUMMARY AND DISCUSSIONS}

In this paper we use the method of QCD sum rules to study light tetraquark states with the exotic quantum number $J^{P C}=3^{-+}$. We find that two quarks and two antiquarks together with at least one derivative are necessary to reach such a quantum number; besides, the quark content can be $q s \bar{q} \bar{s}$ ( $q=$ up/down and $s=$ strange), but cannot be $s s \bar{s} \bar{s}$.

Altogether we have constructed six diquark-antidiquark interpolating currents, where the derivative can only be inside the diquark/antidiquark field, i.e.,

$$
\eta=[q \stackrel{\leftrightarrow}{D} s][\bar{q} \bar{s}]+[q s][\bar{q} \stackrel{\leftrightarrow}{D} \bar{s}]
$$

We use them to perform QCD sum rule analyses, and the results are summarized in Table I. The lowest mass,

$$
M_{\eta_{1}}=2.33_{-0.16}^{+0.19} \mathrm{GeV},
$$

is extracted from the current $\eta_{\alpha_{1} \alpha_{2} \alpha_{3}}^{1}$, which is defined in Eq. (4). From its definition, we clearly see that it contains one "good" diquark of $s_{q s}=1$ and one "good" antidiquark of $s_{\bar{q} \bar{s}}=1$ [51], with one of them orbitally excited:

$$
\left.\mid J^{P C}=3^{-+} ; s_{q s}=s_{\bar{q} \bar{s}}=1 ; l_{q s}=1 \text { or } l_{\bar{q} \bar{s}}=1\right\rangle .
$$

Since the derivative cannot be between the diquark and antidiquark fields, this combination is the most stable one, phenomenologically.

In the present study we have also constructed six meson-meson interpolating currents, as defined in Eqs. (10)-(14). Three of them have the quark combination $[\bar{q} q][\bar{s} s]$, and the derivative is between the two quarkantiquark pairs,

$$
\xi=[\bar{q} q] \stackrel{\leftrightarrow}{D}[\bar{s} s]
$$


the other three have the quark combination $[\bar{q} s][\bar{s} q]$, and the derivative is inside the quark-antiquark pairs,

$$
\xi^{\prime}=[\bar{q} \stackrel{\leftrightarrow}{D} s][\bar{s} q]-[\bar{q} s][\stackrel{\leftrightarrow}{s} q]
$$

Hence, a special decay behavior of the $s q \bar{s} \bar{q}$ tetraquark states with $J^{P C}=3^{-+}$is that (a) they well decay into the $P$-wave $(\bar{q} q)_{S \text {-wave }}(\bar{s} s)_{S \text {-wave }}$ final states but not into the $(\bar{q} q)_{S \text {-wave }}(\bar{s} s)_{P \text {-wave }}$ or $(\bar{q} q)_{P \text {-wave }}(\bar{s} s)_{S \text {-wave }}$ final states, and (b) they well decay into the $(\bar{q} s)_{S \text {-wave }}(\bar{s} q)_{P \text {-wave }}$ final states but not into the $P$-wave $(\bar{q} s)_{S \text {-wave }}(\bar{s} q)_{S \text {-wave }}$ final states.

Especially, we use the Fierz transformation given in Eq. (15) to investigate the light $s q \bar{s} \bar{q}$ tetraquark state defined in Eq. (36). It is well coupled by the current $\eta_{\alpha_{1} \alpha_{2} \alpha_{3}}^{1}$, and its mass has been calculated to be $2.33_{-0.16}^{+0.19} \mathrm{GeV}$. Its isospin can be either $I=0$ or $I=1$, which cannot be differentiated in the present study. It has the following special decay behavior: (a) it decays well into the $P$-wave $\rho \phi / \omega \phi$ channel but not into the $\rho f_{2}(1525) / \omega f_{2}(1525) / \phi f_{2}(1270)$ channels, and (b) it decays well into the $K^{*}(892) \bar{K}_{2}^{*}(1430)$ channel but not into the $P$-wave $K^{*}(892) \bar{K}^{*}(892)$ channel. Note that some of these features can also be derived by analyzing quantum numbers of the initial and final states.

This state lies very close to the $K^{*}(892) \bar{K}_{2}^{*}(1430)$ threshold. Theoretically, it is not so easy to differentiate them, since we do not yet understand the $K_{2}^{*}(1430)$ meson well. However, experimentally, one may be able to do this, since the $K^{*}(892)$ and $K_{2}^{*}(1430)$ mesons are both not very narrow, i.e., $\Gamma_{K^{*}(892)}=50.3 \pm 0.8 \mathrm{MeV}$ and $\Gamma_{K_{2}^{*}(1430)}=98.5 \pm 2.7 \mathrm{MeV}$ [1]. We propose to investigate the $P$-wave $\rho \phi / \omega \phi$ channel in future BESIII, Belle-II, and GlueX experiments. If there existed a narrower resonance of $J^{P C}=3^{-+}$, it would be more likely to be a compact $s q \bar{s} \bar{q}$ tetraquark state other than a $K^{*}(892) \bar{K}_{2}^{*}(1430)$ molecular state. For completeness, in the present study we have also studied its partner state with the quark content $q q \bar{q} \bar{q}$, whose mass is extracted to be $2.27_{-0.17}^{+0.28} \mathrm{GeV}$.

To end this paper, we note that the BESIII Collaboration is possibly able to analyze some of the above decay channels simultaneously. For example, in Ref. [36] they performed a partial-wave analysis for the process $e^{+} e^{-} \rightarrow K^{+} K^{-} \pi^{0} \pi^{0}$. They analysed the four subprocesses $K^{+}(1460) K^{-}, \quad K_{1}^{+}(1400) K^{-}, \quad K_{1}^{+}(1270) K^{-}, \quad$ and $K^{*+}(892) K^{*-}(892)$, where they clearly observed the $\phi(2170) / Y(2175)$ in the former two processes but not in the latter two processes.

\section{ACKNOWLEDGMENTS}

We thank Wen-Biao Yan for useful discussions. This project is supported by the National Natural Science Foundation of China under Grants No. 11722540, No. 12005172, and No. 12075019 and the Fundamental Research Funds for the Central Universities.
[1] C. Patrignani et al. (Particle Data Group), Chin. Phys. C 40, 100001 (2016).

[2] Y. R. Liu, H. X. Chen, W. Chen, X. Liu, and S. L. Zhu, Prog. Part. Nucl. Phys. 107, 237 (2019).

[3] R. F. Lebed, R. E. Mitchell, and E. S. Swanson, Prog. Part. Nucl. Phys. 93, 143 (2017).

[4] A. Esposito, A. Pilloni, and A. D. Polosa, Phys. Rep. 668, 1 (2017).

[5] F. K. Guo, C. Hanhart, U. G. Meißner, Q. Wang, Q. Zhao, and B. S. Zou, Rev. Mod. Phys. 90, 015004 (2018).

[6] A. Ali, J. S. Lange, and S. Stone, Prog. Part. Nucl. Phys. 97, 123 (2017).

[7] S. L. Olsen, T. Skwarnicki, and D. Zieminska, Rev. Mod. Phys. 90, 015003 (2018).

[8] M. Karliner, J. L. Rosner, and T. Skwarnicki, Annu. Rev. Nucl. Part. Sci. 68, 17 (2018).

[9] N. Brambilla, S. Eidelman, C. Hanhart, A. Nefediev, C. P. Shen, C. E. Thomas, A. Vairo, and C. Z. Yuan, Phys. Rep. 873, 1 (2020).

[10] F. K. Guo, X. H. Liu, and S. Sakai, Prog. Part. Nucl. Phys. 112, 103757 (2020).
[11] H. X. Chen, A. Hosaka, and S. L. Zhu, Phys. Rev. D 78, 054017 (2008).

[12] H. X. Chen, A. Hosaka, and S. L. Zhu, Phys. Rev. D 78, 117502 (2008).

[13] C. K. Jiao, W. Chen, H. X. Chen, and S. L. Zhu, Phys. Rev. D 79, 114034 (2009).

[14] Z. R. Huang, W. Chen, T. G. Steele, Z. F. Zhang, and H. Y. Jin, Phys. Rev. D 95, 076017 (2017).

[15] H. J. Lee, New Phys. Sae Mulli 70, 836 (2020).

[16] M. L. Du, W. Chen, X. L. Chen, and S. L. Zhu, Chin. Phys. C 37, 033104 (2013).

[17] Y. C. Fu, Z. R. Huang, Z. F. Zhang, and W. Chen, Phys. Rev. D 99, 014025 (2019).

[18] C. A. Meyer and E. S. Swanson, Prog. Part. Nucl. Phys. 82, 21 (2015).

[19] K. G. Chetyrkin and S. Narison, Phys. Lett. B 485, 145 (2000).

[20] Z. F. Zhang, H. Y. Jin, and T. G. Steele, Chin. Phys. Lett. 31, 051201 (2014).

[21] Z. R. Huang, H. Y. Jin, and Z. F. Zhang, J. High Energy Phys. 04 (2015) 004. 
[22] Z. R. Huang, H. Y. Jin, T. G. Steele, and Z. F. Zhang, Phys. Rev. D 94, 054037 (2016).

[23] J. Ho, R. Berg, W. Chen, D. Harnett, and T. G. Steele, Phys. Rev. D 98, 096020 (2018).

[24] C. F. Qiao and L. Tang, Phys. Rev. Lett. 113, 221601 (2014).

[25] L. Tang and C. F. Qiao, Nucl. Phys. B904, 282 (2016).

[26] A. Pimikov, H. J. Lee, N. Kochelev, P. Zhang, and V. Khandramai, Phys. Rev. D 96, 114024 (2017).

[27] D. R. Thompson et al. (E852 Collaboration), Phys. Rev. Lett. 79, 1630 (1997).

[28] A. Abele et al. (Crystal Barrel Collaboration), Phys. Lett. B 446, 349 (1999).

[29] G. S. Adams et al. (E862 Collaboration), Phys. Lett. B 657, 27 (2007).

[30] M. Ablikim et al. (BESIII Collaboration), Phys. Rev. Lett. 106, 072002 (2011).

[31] J.Z. Bai et al. (BES Collaboration), Phys. Rev. Lett. 91, 022001 (2003).

[32] M. Ablikim et al. (BES Collaboration), Phys. Rev. Lett. 95, 262001 (2005).

[33] M. Ablikim et al. (BESIII Collaboration), Chin. Phys. C 34, 421 (2010).

[34] M. Ablikim et al. (BESIII Collaboration), Eur. Phys. J. C 80, 746 (2020).

[35] M. Ablikim et al. (BESIII Collaboration), Phys. Rev. D 93, 112011 (2016).

[36] M. Ablikim et al. (BESIII Collaboration), Phys. Rev. Lett. 124, 112001 (2020).
[37] E. Kou et al. (Belle-II Collaboration), Prog. Theor. Exp. Phys. 2019, $123 \mathrm{C} 01$ (2019).

[38] A. Austregesilo (GlueX Collaboration), Int. J. Mod. Phys. Conf. Ser. 46, 1860029 (2018).

[39] W. Zhu, Y. R. Liu, and T. Yao, Chin. Phys. C 39, 023101 (2015).

[40] Q. X. Shen, B. A. Li, H. Yu, and M. M. Zhang, High Energy Phys. Nucl. Phys. 8, 573 (1984).

[41] H. X. Chen, X. Liu, A. Hosaka, and S. L. Zhu, Phys. Rev. D 78, 034012 (2008).

[42] H. X. Chen, C. P. Shen, and S. L. Zhu, Phys. Rev. D 98, 014011 (2018).

[43] E. L. Cui, H. M. Yang, H. X. Chen, W. Chen, and C. P. Shen, Eur. Phys. J. C 79, 232 (2019).

[44] K. C. Yang, W. Y. P. Hwang, E. M. Henley, and L. S. Kisslinger, Phys. Rev. D 47, 3001 (1993).

[45] S. Narison, Cambridge Monogr. Part. Phys., Nucl. Phys., Cosmol. 17, 1 (2002).

[46] V. Gimenez, V. Lubicz, F. Mescia, V. Porretti, and J. Reyes, Eur. Phys. J. C 41, 535 (2005).

[47] M. Jamin, Phys. Lett. B 538, 71 (2002).

[48] B. L. Ioffe and K. N. Zyablyuk, Eur. Phys. J. C 27, 229 (2003).

[49] A. A. Ovchinnikov and A. A. Pivovarov, Sov. J. Nucl. Phys. 48, 721 (1988).

[50] J. R. Ellis, E. Gardi, M. Karliner, and M. A. Samuel, Phys. Rev. D 54, 6986 (1996).

[51] R. L. Jaffe, Phys. Rep. 409, 1 (2005). 